\title{
INDEFINITE EXTENSIBILITY IN NATURAL LANGUAGE Pre-print
}

\begin{abstract}
The Monist's call for papers for this issue ended: "if formalism is true, then it must be possible in principle to mechanize meaning in a conscious thinking and language-using machine; if intentionalism is true, no such project is intelligible". We use the GrellingNelson paradox to show that natural language is indefinitely extensible, which has two important consequences: it cannot be formalized and model theoretic semantics, standard for formal languages, is not suitable for it. We also point out that object-object mapping theories of semantics, the usual account for the possibility of non intentional semantics, doesn't seem able to account for the indefinitely extensible productivity of natural language. Keywords: natural language; formalization; indefinite extensibility; universe of discourse; semantics.
\end{abstract}

\section{Introduction.}

There exist at least three published papers (Cook 2007, 2009; Schlenker 2010), arguing that natural language is indefinitely extensible in some sense. More concretely, all the three papers start from the Liar paradox and by means of Strengthened and Strengthened Strengthened Liars construct a hierarchy of truth values with as many of them as there are ordinal numbers. Both authors renounce the principle of Bivalence in order to solve the Liar and its revenge forms.

Bivalence is, however, a fundamental law of classical logic with a considerable intuitive appeal; it is the logical counterpart of the ontological principle that any well-defined situation either obtains or does not obtain. As we see it, trading such a fundamental principle for a solution of the Liar is no bargain. The price could be too high for many people. So, we offer a path to the indefinite extensibility of natural language that is compatible with all principles commonly held as laws of logic. Instead of the Liar, we will use the Grelling-Nelson paradox.

We will first address the issue of formalizability, just to show that natural language is not formalizable. Then we will interpret the result in terms of indefinite extensibility as defined in due time.

\section{Vagueness.}

Some readers may contemplate natural language as trivially non formalizable because of its inherent vagueness. We do not wish to go into the discussion whether vagueness can be formalized or not. We just wish to show that, leaving vagueness aside, natural language turns out to be non formalizable on quite another account, namely, indefinite extensibility.

So we ask the reader to assume we are dealing with a definite segment of English which we will call $\Delta$-English- so that all metalinguistic predicates here used are definite and that the corresponding sets, when they exist, are ordinary nonfuzzy sets. The reader may well not believe such a crisp core of English to exist but this should not prevent him from following us: we only purport to show that on the assumption that $\Delta$-English exists we can all the same prove that it cannot be formalized; this will reveal at least that 
vagueness is not the unique feature of natural language that makes it incapable of being formalized.

\section{Natural language cannot be formalized.}

In order to prove the proposition in the title we first introduce some definitions.

An interpreted language $\mathrm{L}$ is a set of ordered pairs 〈signifier, signified $\rangle$, where a signifier is a syntactic object and a signified is the meaning or semantic value of the signifier (the terms are borrowed from Saussure 1972). For present purposes, we need not commit ourselves to any particular theory of meaning. It is clear that natural language is intrinsically interpreted.

It seems reasonable to require for $\mathrm{L}$ to be formalizable that there be an effective procedure enumerating all of L's signifiers and assigning them the corresponding signified, which amounts to the requirement that $\mathrm{L}$ be recursively enumerable. So we say that an interpreted language L can be formalized if and only if there is an algorithm ALG that enumerates L.

We will establish the following proposition: there is no ALG providing a formalization of the predicates of $\Delta$-English. For the proof, we will use the Grelling-Nelson paradox (Grelling and Nelson 1908) to show that there is no ALG able to enumerate the set of all pairs $\langle$ signifier, signified $\rangle$ that are predicates of $\Delta$-English.

Let $\mathrm{Q}(\mathrm{x})$ be (the crisp core of) the predicate ' $x$ is a predicate of $\Delta$-English'. Since it is a crisp core, $\mathrm{Q}(\mathrm{x})$ is not a vague predicate; hence, it belongs in $\Delta$-English.

If ALG exists, then $\mathrm{L}$ is a set: if $\mathrm{L}$ is recursively enumerable, it is enumerable; hence there is a function from the set $\mathrm{N}$ of all natural numbers onto $\mathrm{L}$; then, by the Axiom of Replacement, $\mathrm{L}$ is a set. Then, since $\mathrm{Q}(\mathrm{x})$ expresses a definite property, we can use that property to separate from L a subset $\mathrm{K}$ containing exactly the members of L satisfying $\mathrm{Q}(\mathrm{x})$. This is just an application of the usual set theoretic Separation axiom. Membership in $\mathrm{K}$ is definite, for $\mathrm{Q}(\mathrm{x})$ is definite.

We will show that $\mathrm{K}$ does not exist.

Assume for reductio that $\mathrm{K}$ exists. Since $\mathrm{K}$ is definable -we have just defined it by means of $\mathrm{L}$ and the definite condition $\mathrm{Q}(\mathrm{x})$-, we can define the predicate $\mathrm{K}_{\mathrm{H}}$ as follows: an expression $P$ is $K_{H}$ if and only if $P$ is in $K$ and $P$ when predicated of $P$ yields a false proposition. Roughly, $K_{H}$ is the predicate ' $x$ is a $\Delta$-English predicate not applying to itself'. Formally:

$$
\forall \mathrm{P}\left(\mathrm{K}_{\mathrm{H}}(\mathrm{P}) \leftrightarrow \mathrm{P} \in \mathrm{K} \& \sim \mathrm{P}(\mathrm{P})\right)
$$

We call $\mathrm{K}_{\mathrm{H}}$ the Grelling-Nelson diagonal predicate of $\mathrm{K}$. More generally, if $\mathrm{S}$ is a set of predicates, $\mathrm{S}_{\mathrm{H}}$ will denote its Grelling-Nelson diagonal predicate hereafter, that is, the predicate applying exactly to those predicates in $\mathrm{S}$ not applying to themselves.

If $\mathrm{K}$ exists, $\mathrm{K}_{\mathrm{H}}$ is definite because, for any object $\mathrm{P}$, either $\mathrm{P}$ is in $\mathrm{K}$ or it is not (for membership in $\mathrm{K}$ is definite), and 
1. If $P$ is in $K$, then $P$ is definite because, by definition of $K$, any predicate in $K$ is an expression of $\Delta$-English. As a consequence, $\mathrm{P}(\mathrm{P})$ has a definite truth value; then, by (1), $\mathrm{K}_{\mathrm{H}}(\mathrm{P})$ has a definite truth value.

2. If $P$ is not in $K$, then, by (1), it is definitely not the case that $K_{H}(P)$.

Consequently, if K exists, $\mathrm{K}_{\mathrm{H}}$ is in $\mathrm{K}$. Then from (1) we have:

$$
\mathrm{K}_{\mathrm{H}}\left(\mathrm{K}_{\mathrm{H}}\right) \leftrightarrow \sim \mathrm{K}_{\mathrm{H}}\left(\mathrm{K}_{\mathrm{H}}\right)
$$

Since (2) is impossible, $\mathrm{K}$ does not exist. Therefore, $\mathrm{L}$ is no set. As a consequence, ALG does not exist and there is no formalization of all $\Delta$-English predicates; since the predicates of $\Delta$-English are a part of English, neither English nor any other sufficiently similar natural language can be formalized.

\section{Indefinite extensibility: natural language goes beyond sethood.}

Once we have obtained our result for a context free of fuzziness, we can set aside all concerns about it and need not speak any more of $\Delta$-English, crisp cores or definite predicates. This will give us a gain in simplicity.

We will use a weakened, set theoretical and simplified version of the formalization of the concept of relative indefinite extensibility by Shapiro and Wright (2006).

We say that concept $C_{1}$ is indefinitely extensible relative to concept $C_{2}$ if and only if for any set $\mathrm{S}$ falling under $\mathrm{C}_{2}$ of objects falling under $\mathrm{C}_{1}$ there is a diagonal object $d$ such that

1. $d$ falls under $\mathrm{C}_{1}$.

2. $d \notin \mathrm{S}$.

3. $\mathrm{S} \cup\{d\}$ falls under $\mathrm{C}_{2}$.

As a consequence, there is no maximal set falling under $\mathrm{C}_{2}$ of objects falling under $\mathrm{C}_{1}$; hence there is no set falling under $\mathrm{C}_{2}$ of all objects falling under $\mathrm{C}_{1}$.

Examples of relative indefinite extensibility are the facts that there is no finite set containing all natural numbers, no enumerable set containing all sets of natural numbers (by Cantor's theorem), no recursively enumerable set containing (the codes of) all arithmetical truths (by Gödel's theorem), no arithmetically definable set containing (the codes of) all arithmetical truths (by Tarski's theorem), simply no set containing all ordinals (by the Burali-Forti paradox), etc.

By 'indefinite extensibility simpliciter of a concept $C$ ' we will mean henceforth any relative indefinite extensibility implying there is no set of all objects falling under $\mathrm{C}$. So we will say that $\mathrm{C}$ is indefinitely extensible if and only if for any set $\mathrm{S}$ of objects falling under $\mathrm{C}$ there is an object $d$ such that

1. $d$ falls under $\mathrm{C}$.

2. $d \notin \mathrm{S}$. 
Applying the notion to our case, we note that the nonexistence of $\mathrm{K}$ in section 3 is due to the fact that the concept of 'English predicate' is indefinitely extensible relative to the concept of 'definable set', which (according to the general definition above) means that, for any definable set $\mathrm{S}$ of English predicates there is a diagonal object $d$ such that

1. $d$ is an English predicate;

2. $d \notin \mathrm{S}$;

3. $S \cup\{d\}$ is a definable set.

Therefore, no maximal definable set of English predicates exists; hence there is no definable set of all English predicates.

So for any definable set $\mathrm{S}$ of English predicates we can use the Grelling-Nelson diagonal predicate of $\mathrm{S}$ to diagonalize out of $\mathrm{S}$ : by letting $d=\mathrm{S}_{\mathrm{H}}$, we can use the Grelling-Nelson paradox to prove the indefinite extensibility of $\mathrm{S}$. This delivers a hierarchy of sets and diagonal predicates:

$$
\mathrm{S}, \mathrm{S}_{1}=\mathrm{S} \cup\left\{\mathrm{S}_{\mathrm{H}}\right\}, \mathrm{S}_{2}=\mathrm{S}_{1} \cup\left\{\mathrm{S}_{1 \mathrm{H}}\right\}, \ldots, \mathrm{S}_{\omega}=\cup \mathrm{S}_{\mathrm{n}}, \ldots
$$

whose indices go through all the definable ordinals (whichever they are).

As said above, the outcome is that there is no definable set of all English predicates, hence, simply no set at all of English predicates, since if that set existed, it would be defined by the expression 'the set of all English predicates'. If such a set existed, the corresponding diagonal predicate would be a self-diagonalizer, i.e. a diagonalizer diagonalizing out of a multiplicity of which it is itself an element (the concept is taken from Luna and Small 2009). That there are no such diagonalizers is established by this version of Thomson's small theorem (Thomson 1962):

$$
\sim \exists \mathrm{x}(\mathrm{x} \in \mathrm{S} \& \forall \mathrm{y}(\mathrm{Rxy} \leftrightarrow \mathrm{y} \in \mathrm{S} \& \sim \mathrm{Ryy}))
$$

that is, no set $\mathrm{S}$ contains a member $x$ having the relation $\mathrm{R}$ exactly with those members of $S$ that do not have the relation with themselves. It is easy to see that $\mathrm{K}_{\mathrm{H}}$ would be such an $x$ if $\mathrm{K}$ existed: it would be in $\mathrm{K}$ and would apply to just those objects in $\mathrm{K}$ that do not apply to themselves.

It seems, however, that we do not need paradoxes to show that the (concept of) English language is indefinitely extensible. English, as any other natural language, can surely be represented as an improper one-to-many function $f$ mapping syntactic objects to meanings, that is to say, mapping signifiers to signifieds. English expressions are not just signifiers but ordered pairs 〈signifier, signified $\rangle$ in $f$. Now there can be no English syntactic object $x$ such that $f(x)=f$, because no function, considered as a set of ordered pairs can be a member of one of its members; the axiom of Foundation of usual set theory stays in the way by prohibiting any set from being a member of itself or a member of one of its members, etc. This means that we do not have in English a name for English and, in general, there can be no name able to refer to the whole language of which it is a member. When we name English in English, the named English (the object-language) does not contain the metalinguistic expression 'English' we are just using, although it may contain its signifier with a more restricted applicability (Luna 
and Taylor 2010). As a consequence, we can produce an English expression beyond any nameable set of English expressions and in fact the expression 'English language' diagonalizes out of whatever it is able to denote.

If we consider in addition that any item in the metalanguage can be turned into a new item of an extended object-language, we find in this hierarchy of language levels, required to avoid circularity, a root of indefinite extensibility in natural language.

\section{Semantics, quantifiers, and the cardinality problem.}

The hierarchy of language levels is the natural way out of the linguistic paradoxes such as the Grelling-Nelson and the definability paradoxes: Richard's and Berry's, for instance. But there are two circumstances we want to emphasize: that the hierarchy of extensibility levels goes beyond sethood and that it is semantics that provokes indefinite extensibility in natural language.

Obviously, mere symbol handling can in no way bring it about. Regarding the proposition in section 3 some readers may have wondered what would happen if an algorithm ALG* generating English phrases outputted the phrase 'is an English predicate outputted by $A L G^{*}$ and it yields a false proposition when predicated of itself", that is to say, if the set generated by ALG* contained the signifier of its own Grelling-Nelson diagonal predicate. Could a situation like this contradict the assertion that $\mathrm{K}_{\mathrm{H}}$ cannot be in $\mathrm{K}$ in the proposition's proof?

Certainly such algorithms exist but, of course, this fact by no means proves the proof faulty. In fact, ALG*'s diagonal predicate as generated by $\mathrm{ALG}^{*}$ can be attached no definite meaning: it is meaningless at the language level it is generated on; any mapping procedure that could assign to the members of the output of $\mathrm{ALG}^{*}$ their corresponding meanings in English would render the diagonal object a paradoxical expression, not a predicate; it would just reproduce the Grelling-Nelson paradox.

Thus the reason why the set $\mathrm{K}$ in section 3 cannot exist is that the required interpretation cannot exist: there is no function assigning meanings to any set of syntactical objects able to exhaust the meanings of all natural language predicates. If such a function existed, we could use the set theoretic axiom of Replacement to show that the collection of natural language predicates is set-sized, which we have seen it is not. The axiom of Replacement prevents any function from being onto a collection that is not a set. Thus, what renders natural language non formalizable is the impossibility of a set-sized bridge (i.e. a function) between syntax and semantics, let alone a recursive or recursively enumerable one.

In order to see that syntax cannot go all the way up to indefinite extensibility, we can also consider that syntax can take us as far as automata can and, under Church's thesis, this is no farther than the least non Church-Kleene-constructive ordinal $\omega_{1}{ }^{\mathrm{CK}}$ (Church 1936; Church and Kleene 1936; Kleene 1938). On the contrary, definable ordinals and language extensibility levels go indefinitely beyond: to see this, simply note that we can name $\omega_{1}{ }^{\mathrm{CK}}$ and this enables us to ride on our diagonalization procedures beyond it.

It is again semantics that hands us the solution of the cardinality problem. This problem is posed by the fact that the set of English expressions seems unable to exceed the 
countable; the reason is rather obvious: finite symbol strings over a finite alphabet can at most make a countably infinite set. And the reason why this is a problem is that if there were a bijection between the natural numbers and such expressions, the axiom of Replacement would surely convince us that such expressions form a set, which they do not.

Luna and Taylor (2010) have addressed the cardinality problem in the context of the Richard paradox. It seems the problem can be solved by resorting to the Saussurian distinction between the syntactic components or signifiers of natural language expressions (which add up, indeed, to a countable set) and the expressions themselves, which should be regarded as pairs 〈signifier, signified〉 of a syntactic element coupled with a semantic one. The point is that there may not be an injection of the expressions onto the signifiers, that is, in the usual jargon, there might be more expressions than signifiers. But this can only happen if some signifiers are associated in natural language with more than one signified (otherwise, there would be an obvious injection from the expressions to the signifiers). And that ambiguity of the signifiers should be essential in some sense, that is to say, forced upon natural language by the same essential features that make it indefinitely extensible. Let's examine how the required ambiguity could arise.

At any level of language extensibility which we can reach by means of Grelling-Nelson diagonal objects, there is a set $\mathrm{S}$ of all the English predicates existing on that level. Then the Grelling-Nelson diagonal predicate $S_{\mathrm{H}}$ can be standardly constructed (by means of the concept English predicate) as

$\left(\mathrm{S}_{\mathrm{H}}\right) \quad$ is an English predicate and it yields a false proposition when predicated of itself'

Since the concept of English predicate has variable extension along the hierarchy of extensibility levels, the signifier of $S_{H}$ signifies differently along that hierarchy, and this furnishes an infinite variety of meanings for this signifier. The reader may be thinking now that the ambiguity in $\left(\mathrm{S}_{\mathrm{H}}\right)$ above can be avoided by turning it into something like:

$\left(\mathrm{S}_{\mathrm{H} \lambda}\right)$ 'is an English predicate at language level $\lambda$ and it yields a false proposition when predicated of itself'

for each language level $\lambda$.

And the reader may be thinking also that the paradox can be regained by means of an expression like:

$\left(\mathrm{S}_{\mathrm{H} \Omega}\right)$ 'is an English predicate at some language level $\lambda$ and it yields a false proposition when predicated of itself'

In order to address the first objection, we need only point out that we would never have the syntactic resources to name without ambiguity all language levels, since these levels go beyond sethood, hence beyond the countable, whereas the syntactic resources of any language containing only finitely long formulas stop at the countably infinite. 
In order to address the second objection, we argue that any attempt at neutralizing level equivocation in the Grelling-Nelson diagonal predicate would confront the impossibility of quantifying over all the extensibility levels of language or, equivalently, over all the definable ordinals. The quantifier in 'some extensibility level' means differently along the levels of natural language extensibility.

In standard model theory the quantifiers of a formal language are only allowed to range over a (non empty) set. The rationale for this is the following: we can only refer at once to all objects in a multiplicity if those objects can all be simultaneously given; and, surely, when this condition is fulfilled the multiplicity can be regarded as a single object and this seems to mean precisely that the multiplicity is a set. According to this, when by means of 'all', 'any', 'some', etc. we quantify over the extension of an extensible concept, our quantifiers mean variously along the extensibility levels because their domain expands as it is used at higher and higher levels. ${ }^{1}$

Say that a quantifier is bounded by a predicate $P$ if and only if it heads an expression of the form ' $\forall \mathrm{x}(\mathrm{Px} \rightarrow \ldots)$ ' or ' $\exists \mathrm{x}$ (Px \& ...)'. Assume the usual model theoretic assumption that only (non empty) sets are possible universes of discourse (i.e. domains of quantification) and consider an expression where the universal quantifier is bounded by a predicate corresponding to a concept that is indefinitely extensible. Then the range of the quantifiers in that expression varies along the unbounded hierarchy of extensibility levels: at each level we have a set of P's over which it is possible to quantify, though the set of all P's across all levels does not exist.

Such is the case for the quantifier in the phrase 'all English predicative expressions' but also for the quantifier in 'some extensibility level' because these levels make up no set: if they formed a set, the usual set theoretical axiom of Union would give us the set of all the items across all the extensibility levels of an indefinitely extensible concept, in contradiction with the assumption that the concept is indefinitely extensible.

The ambiguity of the quantifier in the expression 'some extensibility level' makes the concept of extensibility level itself indefinitely extensible. So, we cannot unambiguously refer to all extensibility levels of natural language: the range of our reference would become ever wider along the extensibility stages without ever coming to an ultimate extension. There is no way to get around this ambiguity: it is the essential ambiguity we were after.

The claim that our universe of discourse widens along the extensibility levels we will call 'indefinite extensibility of the universe of discourse', for short IEUD.

Now some readers may be concerned about the seemingly disastrous consequences of this use of standard model theoretic semantics: if there are objects (like ordinals or extensibility levels) such that we cannot refer to all of them at the same time, what about propositions like 'all ordinals are transitive'? For this problem I refer the reader to Luna 2008. It is argued there, following hints by Carnap and Gödel, that such universal

\footnotetext{
${ }^{1}$ However, the claim, suggested by standard model theoretic semantics, that indefinite extensibility somehow implies the impossibility of absolutely unrestricted quantification is disputed in the monographic 'Absolute Generality' (Rayo, Uzquiano 2006). Some authors (e.g. Glanzberg, Parsons, Hellman) tend to endorse it while others (e.g. Rayo, Williamson, Linnebo) explore ways to get around it.
} 
quantifiers are apparent quantifiers, meaning that they do not have extensional but intensional nature, that is, that they do not represent reference to all members of a non set-sized extension but intensional entailment: the proposition 'all ordinals are transitive' ought to be read as 'the concept of ordinal entails the feature of being a transitive set'.

The reader should not overlook the remarkable fact that combining the notion of indefinite extensibility with the model theoretical equation of domain of quantification with (non empty) set, we get the means to cope not only with the Grelling-Nelson paradox (and other paradoxes of natural language) but also with the cardinality problem, for it is the concept of indefinite extensibility we use to solve the former that provides the source of the ambiguity in natural language required to solve the latter: since our quantifiers can only quantify over sets and indefinite extensibility takes us beyond sethood, our quantifiers are ambiguous in that their ranges expand as we pass from one extensibility level to another. This reveals some serendipity together with some internal economy in the theory.

\section{Object-object mappings or intentional states?}

The Monist's call for papers for this issue proposes the following question:

Purely formal semantic approaches, including computer modelings, treat meaning as an abstract mapping of objects onto objects, with no reference to a thinker expressing thoughts by means of language. An intentionalist theory, in contrast, considers expressive intentional states as essential to understanding meaning, but opens itself in turn to a number of objections.

In order to say a word on this issue, it is natural to ask how indefinite extensibility, and particularly IEUD, impacts the semantics of natural language. After all, it is semantics that drives natural language beyond sethood into the indefinitely extensible.

It is an immediate consequence of IEUD that the model theoretic semantics, which is standard for formalized languages, is not appropriate for natural language. Standard model theoretic starts off with the provision of a non empty set as a fixed universe of discourse or domain of quantification. But, as we have seen, there is no set that could be proposed as the universe of discourse of natural language. The possibility of diagonalizing indefinitely out of any given set of natural language predicates, as revealed by the Grelling-Nelson paradox, suggests that the range of the quantifiers in natural language is never ultimately fixed; indeed, it intimates that the range of the quantifiers in natural language grows ever wider along the extensibility levels (see for a more detailed treatment of this point Luna and Taylor 2010, appendix).

Let's take a look once more at the dynamics of indefinite extensibility in natural language.

Assume that the universe of discourse of natural language at a particular extensibility level is one fixed set $\mathrm{S}$. We can then define the Grelling-Nelson diagonal predicate $\mathrm{S}_{\mathrm{H}}$ for $S$ by just quantifying over all predicative expressions in $S$. In doing so, we extend our universe of discourse from $S$ to $S *=S \cup\left\{S_{H}\right\}$. In fact, we can then state an apparent counterexample to Thomson's theorem:

$$
\exists \mathrm{x}(\mathrm{Px} \& \forall \mathrm{y}(\mathrm{Px} \rightarrow(\mathrm{Rxy} \leftrightarrow \sim \mathrm{Ryy})))
$$


read as 'there is a predicative expression that applies to all predicative expressions that do not apply to themselves', if we understand that ' $\forall \mathrm{y}$ ' in (4) ranges over $S$ while ' $\exists x$ ' ranges over $S^{*}-S=\left\{S_{H}\right\}$, for if so, the new predicate is not an instance for ' $\forall y^{\prime}$ in (4) and this prevents paradox from arising.

On the assumption that every domain of quantification is a set, we can in fact go beyond any universe of discourse just by taking the universe at issue as a set, that is, as a completed whole. Since no set is self-membered, the set will not be in the previous universe of discourse; it would rather diagonalize out of it. We can see this procedure at work in the construction of the set theoretic ordinals, where the successor of an ordinal $\alpha$ is $\alpha \cup\{\alpha\}$.

The indefinite extensibility of the set theoretical ordinals has been proposed as the standard of any indefinite extensibility (Shapiro and Wright 2006). Indeed, the sequence of ordinals is the simplest mathematical representation of the unbounded plus ultra of thought, of its movement beyond whatever it has previously produced. And, what matters most when dealing with natural language, also the definable ordinals are indefinitely extensible. To see this, we only need to use a 'definable' version of the Burali-Forti paradox. Assume there is a set $\Omega_{\mathrm{D}}$ of all definable ordinals and remember that an ordinal is the set of all ordinals less than it; obviously, $\Omega_{\mathrm{D}}$ is definable, if it exists, and it is a well-ordered, well-founded, transitive set, hence a definable ordinal; but $\Omega_{\mathrm{D}}$ is not in $\Omega_{\mathrm{D}}$ because no ordinal is self-membered; so we have produced a definable ordinal that is not in $\Omega_{\mathrm{D}}$, contradicting the assumption it was the set of all definable ordinals, and we have done so just by gathering all the previous ordinals into one new ordinal. It is in fact possible to diagonalize out of any definable set $S$ of definable ordinals by just taking the great union $\cup S$ of $S$ (i.e. the set of the members of the members of $S$ ); it is easy to prove from the usual axioms of set theory that $\cup S$ would be a definable ordinal not in $\mathrm{S}$. This furnishes an indefinitely iterable diagonalization procedure that is akin to the capability of producing the Grelling-Nelson diagonal predicate for any definable set of natural language predicates.

This is a fantastic semantics-driven dynamical scenario. And it poses a problem for any object-object mapping theory of natural language semantics. The re-utilization of signifiers due to the shifting meaning of quantifiers along the extensibility levels would make any mapping dependent on the extensibility level: the mapping would not take a symbol onto an object but a pair 〈symbol, level〉onto an object. Now it seems we can only account for the emergence of levels by bringing in semantics itself; as a consequence, we cannot use levels to account for semantics if we are to avoid circularity. In fact, it seems we can only explain the emergence of language extensibility levels by bringing in the productivity of thought, that is, by resorting to the capability of thought to produce new thought objects via diagonalization. So, in order to produce what accounts for the semantics of the indefinitely extensible natural language, we need not only map signifiers onto signifieds (e.g. names onto ordinals), we need to produce the latter as objects of thought.

The new signifieds are not to be found in the physical environment; they are abstract objects that must be generated by reflection on what has already been produced and by diagonalization out of it; but reflection on and diagonalization out of can only be conceived as operations of intentional thought; it is hard to even try to figure out what 
else they could be. Thus, for all we know, including in the picture the productivity of intentional thought, its capability of diagonalization, is intuitively necessary to account for the extension beyond sethood of the definable ordinals. And this seems to imply that intentionality theories of meaning are more suitable for IEUD than any theory devised to the effect of rendering semantics possible for mechanical, non intentional linguistic behavior.

\section{Conclusions.}

Paradoxes such as the Grelling-Nelson reveal a significant feature of natural language, namely, its indefinite extensibility. This feature implies both that natural language cannot be formalized and that the standard semantics for formal languages are not suitable for natural language. The phenomenon of indefinite extensibility reveals a radical divide both between natural and formal languages and between their respective semantics.

Since indefinite extensibility in natural language is a semantic phenomenon based on the possibility of re-utilizing the same syntactic resources to denote something new in a new logical context (i.e. a new extensibility level), and this in turn is only possible due to the production of new thought objects via diagonalization, there is a need to invoke the productivity of thought to account for that extensibility. The need to invoke thought contents in the explanation of the indefinite extensibility of natural language suggests that no non intentional account of natural language semantics could be adequate.

\section{REFERENCES}

Church, A. (1936). The constructive second number class. Bulletin of the American Mathematical Society, 44, 224-232.

Church, A. \& Kleene, S. C. (1936). Formal definitions in the theory of ordinal numbers. Fundamenta Mathematicae, 28, 11-21.

Cook, R. T. (2007). Embracing the revenge: on the indefinite extensibility of language. (In J.C. Beall (Ed.), Revenge of the Liar (pp. 31-52). Oxford: Oxford University Press.)

Cook, R. T. (2009). What is a truth value and how many are there? Studia Logica 92, 183-201.

Glanzberg, M. (2006). Context and unrestricted quantification. (In A. Rayo \& G. Uzquiano (Eds.), Absolute Generality (pp. 45-74). Oxford: Oxford University Press.)

Grelling, K. \& Nelson, L. (1908). Bemerkungen zu den Paradoxien von Russell und Burali-Forti. Abhandlungen der Fries'schen Schule (new series), 2, 301-334.

Kleene, S. C. (1938). On notations for ordinal numbers. The Journal of Symbolic Logic, 3(4), 150-155.

Luna, L. (2008). Can we consistently say that we cannot speak about everything? The Reasoner, 2(9), 5-7. 
Luna, L. \& Small, C. (2009). Intentionality and computationalism. A diagonal argument. Mind and Matter, 7(1), 81-90.

Luna, L. \& Taylor, W. (2010). Cantor's proof in the full definable universe. Australasian Journal of Logic, 8, 10-25.

Saussure, F. (1972). Cours de Linguistique Générale. (Paris: Payot.)

Schlenker, P. (2010). Super Liars. The Review of Symbolic Logic 3, 374-414.

Shapiro, S. \& Wright, C. (2006). All things indefinitely extensible. (In A. Rayo \& G. Uzquiano (Eds.), Absolute Generality (pp. 255-304). Oxford: Oxford University Press.)

Thomson, J. F. (1962). On some paradoxes. (In R. J. Butler (Ed.), Analytical Philosophy (pp. 104-119). New York: Barnes and Noble.) 